\title{
DINAMIZACIÓN DE LOS BARRIOS HISTÓRICOS HACIA DESTINOS DE TURISMO EXPERIENCIAL. PAPEL DE LAS REDES DE COLABORACIÓN EN LA ESTIMULACIÓN DEL BARRIO DE LAS LETRAS EN MADRID Y BARRIO ITALIA EN SANTIAGO DE CHILE
}

\author{
Stefania PARETI (stefania.pareti@edu.uah.es) \\ Doctorada en Economía y Gestión Empresarial. \\ Blanca GARCÍA HENCHE (blanca.garcia@uah.es) \\ Universidad de Alcalá de Henares. \\ Erica SALVAJ (esalvaj@udd.cl) \\ Universidad del Desarrollo. Santiago de Chile.
}

Recibido: 30 de noviembre de 2018

Aceptado: 10 de diciembre de 2018

RESUMEN: El patrimonio cultural ha comenzado a implementar nuevas estrategias, debido a la extensa proliferación del turismo urbano a nivel internacional. Es por ello que surge la necesidad de potenciar y entender cómo los barrios históricos, al ser intervenidos estratégicamente, pueden resultar ser destinos de turismo experiencial y de ocio diferenciados, poniendo en valor recursos y servicios que a su vez mejoran la vida de la población local, entregando mayor competitividad y sostenibilidad económica y social. El objetivo de este trabajo es explorar y describir cómo el fortalecimiento del asociacionismo y las redes entre quiénes componen la oferta cultural, comercial y turística de los barrios históricos resulta fundamental para la transformación y dinamización de los mismos. Es por esto que se ha seleccionado dos barrios históricos como Barrio Italia (Santiago de Chile) y Barrio de las Letras (Madrid). Dichos barrios son barrios históricos singulares y con personalidad gracias a recursos culturales, etnográficos y comerciales que les hacen únicos. Se llevará a cabo un análisis exploratorio para comprender el asociacionismo y colaboración de los comercios y entidades culturales de cada barrio a través de la metodología de redes, proponiendo a su vez estrategias de marketing y economía de la experiencia. Se concluye y entrega como resultado principal una evidente ubicación de cada barrio en fases diferentes de desarrollo en su ciclo de vida como destino turístico, Barrio Italia se encuentra en una fase incipiente de crecimiento frente a Barrio de las Letras que ya cuenta con posicionamiento turístico más consolidado.

PALABRAS CLAVE: Teoría de Redes, Marketing, Barrios Históricos, Barrios “destino turístico", Turismo Cultural 
DYNAMIZATION OF THE HISTORICAL NEIGHBORHOODS TOWARDS EXPERIENCIAL TOURISM DESTINATIONS. ROLE OF COLLABORATION NETWORKS IN THE STIMULATION OF THE NEIGHBORHOOD OF LETTERS IN MADRID AND BARRIO ITALY IN SANTIAGO DE CHILE

ABSTRACT: Cultural heritage has begun to implement new strategies, due to the widespread proliferation of urban tourism internationally. That is why the need arises to enhance and understand how the historical, strategic services, tourism destinations, experience and leisure, value and resources, and the best way to improve the lives of the local population, delivering greater competitiveness and economic and social sustainability. The objective of this work is to explore and describe how the strengthening of associations and networks among those who make up the cultural, commercial and tourist offer of historic districts is fundamental for the transformation and revitalization of them. This is why it has been selected two historical districts as Barrio Italia (Santiago de Chile) and Barrio de las Letras (Madrid). These districts are unique historical districts with cultural, ethnographic and commercial resources that make them unique. An exploratory analysis will be carried out to understand the associationism and collaboration of the businesses and cultural entities of each district through the network methodology, proposing in turn marketing strategies and experience economy. The study concludes and delivers as a main result an evident location of each district in different phases of development in its life cycle as a tourist destination, Barrio Italia is in an incipient phase of growth in front of Barrio de las Letras that already has tourist positioning more consolidated.

KEYWORDS: Assosiationism, Network Theory, Marketing, Cultural District, Destination neighborhoods, Cultural Tourism

\section{INTRODUCCIÓN}

El patrimonio cultural posee un papel de dinamizador socioeconómico y favorece el desarrollo local a través de la explotación del turismo cultural. Pero, si el patrimonio va más allá de lo puramente cultural, transformándose en un potencial recurso económico, debe ser protegido para garantizar su continuidad y sostenibilidad (Camarero y Garrido, 2004). Fortalecer el asociacionismo y las redes entre quiénes componen la oferta cultural, comercial y turística de barrios históricos de las ciudades con una fuerte oferta de turismo cultural, resulta fundamental para el desarrollo y sustentabilidad urbana de los mismos.

El objetivo de este estudio es conseguir un acercamiento a esta realidad en el caso de la ciudades de Madrid y Santiago de Chile, donde determinados barrios se convierten en espacios que reivindican el estilo de vida local y la singularidad como experiencia turística para ese consumidor experiencial que busca alejarse de ese turismo masivo que amenaza los negocios tradicionales en la era de la globalización. El trabajo forma parte de una investigación más amplia donde se han seleccionado esos dos casos de estudio por encontrarse en diferentes fases de ciclo de vida respecto a su desarrollo urbano como destino turístico. 
Los bienes patrimoniales y territorio por sí solos no tienen ningún valor si no existe una red de apoyo que los sustente y hago vivos. Es por ello que se han seleccionado como destinos estratégicos de investigación a Barrio Italia en Santiago de Chile y Barrio de Las Letras en Madrid, considerados como exponentes representativos de la integración entre bienes culturales y territorio. Dichos barrios poseen una dinámica actividad de emprendimiento artístico-cultural y un cuidado comercio local, tradicional y de proximidad, como estrategias de singularización de los mismos.

Por otra parte, se han seleccionado el Barrio de las Letras en Madrid y el Barrio Italia en Santiago de Chile debido a que se encuentran en situaciones muy diferentes en cuanto a al desarrollo del ciclo de vida como producto turístico

En este contexto, se propone como objetivo principal explorar cómo la asociación de pequeñas empresas y las redes creadas entre los miembros asociados permiten la transformación de una zona comercial, creando una imagen de destino y aumentando el valor turístico del territorio a través de la puesta en valor del patrimonio histórico (material e inmaterial), cultural y social de dichos barrios.

A través del estudio realizado en el Barrio de Las Letras en Madrid y Barrio Italia en Santiago de Chile, se explorará cómo el asociacionismo y las redes generan una sinergia entre las partes que lo conforman, proponiendo beneficios sociales y psicológicos en la identidad de quiénes lo componen y a su vez en la percepción de quiénes lo visitan, dándole un sello único e imposible de replicar debida a la diferenciación e imagen de marca desarrollada.

Dicha marca se utilizará como instrumento de comercialización y posicionamiento turístico, ya que la oferta cultural, comercial y de ocio del Barrio de las Letras y de Barrio Italia supone una adaptación y remodelación de un casco histórico que oferta nuevas experiencias a los turistas con nuevas necesidades y, a todo ello, ha contribuido el asociacionismo y las redes de colaboración del pequeño comercio emprendedor de la zona creando marcas identificativas de destino, junto con instituciones culturales ubicadas en dicho barrio

La justificación de la presente investigación, se sustenta con que no existe a la fecha metodologías sistemáticas para gestionar adecuadamente patrimonio cultural junto a desarrollo territorial y urbano sustentable (Bandarin y Van Oers, 2012).

\section{FUNDAMENTACIÓN TEÓRICA}

El turismo al ser considerado a su vez una actividad con contenido social, ha generado amplias aristas de desarrollo en cuanto al concepto de productos turís- 
ticos (Barbieri, Almeida Santos y Katsube, 2012; McGehee y Andereck, 2009; Wearing, 2001). Es por esto que el modelo tradicional de turismo genera nuevas oportunidades y desarrollos debido a un consumidor cada vez es más exigente y en busca experiencias únicas. Es así como surge en turismo experiencial o emocional (G. Henche, 2017).

Ya a finales de los años 90 Jun Song, Lee, Park, Hwang y Reisinger (2015) y Schmitt (1999) consideran el turismo experiencial como un tema relevante para objeto de estudio en el ámbito del comportamiento del consumidor.

Actualmente se observa que el turismo ha sufrido cambios significativos, si se compara con la década de los años 60 cuándo el turismo de masa era la tendencia predominante y era incluso considerada como lujo. En la actualidad, en cambio, el consumidor busca ser protagonista de su propia experiencia (Hall y Weiler, 1992; Lo y Lee, 2011; Robinson y Novelli, 2005).

En este nuevo modo de interacción con el turista, los productos y servicios ofrecidos buscan una interacción directa, humana y personalizada, ya que se trata de integrar elementos culturales, intentando hacer vivir las tradiciones del destino, con el fin de conseguir la generación emociones positivas (G.Henche y Salvaj, 2017; Molina, Martín Consuegra, Esteban y Díaz, 2007).

No se ha de olvidar que, en turismo, no se venden realmente productos, sino que se venden experiencias (Vogeler y Hernández, 2002). El turista que busca experiencias es aquel que quiere evadirse, descubrir, disfrutar y conectar con la gente del lugar y con sus costumbres; por ello, los barrios históricos y con personalidad pueden, y deben, ser parte de esa experiencia buscada por los turistas. En ese sentido, España tiene una gran oferta de productos a ofrecer en muy diferentes destinos.

Los denominados "turistas de última o tercera generación", según las últimas tendencias de la demanda, aprovechan su estancia en los destinos urbanos para buscar experiencias enriquecedoras y vivencias únicas. Así, se pasa de un turista pasivo, mero espectador a un turista activo, que en lugar de "observar" se desplaza al destino para "hacer" algo, involucrándose más en la cultura local.

La “economía de la experiencia” (Pine y Gilmore, 1998) lleva a las ciudades a convertirse en escenarios en los que se crean experiencias. En esta economía de la experiencia, la cultura se convierte en una materia prima esencial y el turismo cultural es un elemento cada vez más importante, más aún en las ciudades que poseen un rico patrimonio cultural. 
Este patrimonio cultural se representa tanto en materiales tangibles (museos, catedrales, palacios...) fácilmente enumerados, como en materiales intangibles (tradiciones, gastronomía, estilos de vida, paisajes...) difíciles de enumerar, pero que dan un gran apoyo a la simbología de cualquier ciudad y consolidan su imagen turística (Toitiño, 1996). Por tanto, cuando se habla de patrimonio cultural se ha de incorporar además del patrimonio histórico, otras manifestaciones como la lengua, la artesanía, la gastronomía, etc. Y, es aquí, donde los barrios analizados, en Madrid y Santiago de Chile, se posicionan como patrimonio intangible de la cultura de un territorio.

Comprender la experiencia turística, especialmente en su dimensión sensorial (Schmitt, 1999), es relevante para la optimización de los destinos turísticos. En consecuencia, se han de desarrollar productos innovadores, un entorno atractivo y experiencias que dejen una marca positiva para quien visita el destino, además de contribuir positivamente a la calidad de vida de la comunidad local (Agapito et al., 2013, 2014; Kastenholz et al., 2012).

Algunas de las dimensiones de la autenticidad percibida por los turistas (Wang, 1999) son la autenticidad objetiva, referida a la singularidad de los recursos patrimoniales y territoriales que sirven de soporte a los productos turísticos y la autenticidad constructiva, que hace referencia a la construcción social sobre lo que se considera que tiene valor y atractivo turístico. En este sentido, los barrios seleccionados, Barrio de las Letras en Madrid y Barrio Italia en Santiago de Chile, constituyen un recurso turístico que los nuevos perfiles de consumidores valoran como recursos que aportan autenticidad objetiva y constructiva a los destinos.

King (2002) desarrolla la idea de re-organizar el marketing de los destinos, ya que ya no resulta necesario promocionar los lugares como se hacía años atrás, ahora es necesario lograr generar experiencias que realmente conecten al consumidor con ello. Kotler y Gertner (2002) desarrollan el marketing de lugar junto a una perspectiva de gestión de marca. La imagen de marca de un destino puede definirse como la interpretación subjetiva de un lugar realizada por el turista (Bigné, Sánchez y Sánchez, 2001).

La cooperación y las redes son un verdadero multiplicador de oportunidades en el sector del turismo (Mendonça, Varajão y Oliveira, 2015), ya que ayudan a potenciar la transferencia y fortalecimiento de conocimiento (Zach y Hill, 2017). Así, las redes sociales resultan ser fundamentales para coordinar y organizar la actividad económica (G. Henche, 2017; Gabbay y Zuckerman, 1998; Nahapiet y Ghoshal, 1998). 
El trabajo asociacionista y cooperativo resulta fundamental para generar y consolidar una imagen de destino. La identidad de un lugar, junto a la eficiencia de los emprendedores que proliferan en el lugar y soportan las influencias que se generan y existen en la comunidad, son fundamentales para crear un sistema que se mantenga en el tiempo (Hallak, Brown y Lindsay, 2012).

Es por ello que la cooperación y el asociacionismo resultan ser una alternativa a poder fortalecer una imagen de barrio que ayude a su vez a cada una de los pequeños comercios que lo conforman, lo cual resultaría mucho más complejo de no existir dicha red de colaboración y proximidad. En los barrios de turismo culturales, se generan acciones colaborativas dónde los mismos miembros del barrio se escogen como proveedores como ocurre por ejemplo entre un pequeño local que comercializa cacao y café, se lo vende al café del mismo barrio, todo lo cual se traduce finalmente en la mantención de valores culturales de los barrios históricos.

Por otra parte, el ciclo de vida del destino turístico (CVDT) constituye uno de los temas de mayor relevancia en los análisis de competitividad turística. Muestra la evolución de un destino en el tiempo y, aunque con ciertas limitaciones, puede ayudar a identificar la estrategia más coherente a llevar a cabo.

La utilización del concepto de "Ciclo de Vida del Producto" es bastante reciente en el sector turístico y se ha aplicado especialmente a los destinos turísticos (Serra, 2011). El ciclo de vida del destino turístico constituye uno de los temas de mayor relevancia en los análisis de competitividad turística. El modelo propuesto por Butler en 1980 representa uno de los paradigmas esenciales en torno al desarrollo de los destinos turísticos (Lundtorp y Wanhill, 2001) y es uno de los enfoques de mayor influencia y más ampliamente referenciado en el análisis del desarrollo del turismo local (Harrison, 1995).

Partiendo de que los destinos turísticos son dinámicos y evolucionan en el tiempo, esa evolución o cambio puede ser debida a cambios en los consumidores turísticos (Cohen, 1972; Plog, 1974) o en el propio destino (Christaller, 1964). Ante esa evolución, las empresas y destinos turísticos utilizaran de distinto modo y con distinta intensidad los instrumentos comerciales. Así, es evidente que las acciones de marketing han de adaptarse a la situación o fase del ciclo de vida en que se encuentren los destinos. La evolución condiciona el desarrollo de nuevos productos y la estrategia comercial a seguir. Las estrategias de comunicación, por ello, serán muy diferentes cuando la finalidad de las mismas sea dar a conocer un destino turístico, en las primeras fases del ciclo, que en las fases de consolidación o estancamiento, donde la comunicación estará basada en la imagen de diversificación del producto. 


\section{METODOLOGÍA}

Para la realización de esta investigación, se han utilizado fuentes secundarias y primarias. En primer lugar, se revisan las principales acepciones del asociacionismo en la gestión urbana. También, se estudian los principales cambios del sector turístico urbano hacia un turismo experiencial. Posteriormente, se ha recopilado información secundaria sobre la historia de los barrios y sus asociaciones de comerciantes. Finalmente, se ha recurrido a fuentes primarias de información, para así obtener de primera mano, datos sobre las características de la oferta cultural, comercial y turística.

La investigación primaria cualitativa se basa en entrevistas realizadas a miembros del directorio de la Asociación de Comerciantes de Barrio Italia y Barrio de las Letras, tal como recoge la tabla 1.

Tabla 1: Ficha técnica de la investigación primaria.

\begin{tabular}{|c|c|c|}
\hline & $\begin{array}{l}\text { BARRIO DE LAS LETRAS } \\
\text { (Madrid) }\end{array}$ & $\begin{array}{l}\text { BARRIO ITALIA } \\
\text { (Santiago de Chile) }\end{array}$ \\
\hline Universo & $\begin{array}{l}\text { Unidades comerciales del Barrio de } \\
\text { las Letras. }\end{array}$ & $\begin{array}{l}\text { Unidades comerciales del Barrio } \\
\text { Italia, Santiago de Chile. }\end{array}$ \\
\hline Área Geográfica & Madrid, España. & Santiago, Chile. \\
\hline Muestra & $\begin{array}{l}187 \text { encuestas válidas (de los } 301 \\
\text { comercios del barrio). }\end{array}$ & $\begin{array}{l}198 \text { encuestas válidas (de los } 265 \\
\text { comercios del barrio). }\end{array}$ \\
\hline $\begin{array}{l}\text { Procedimiento de } \\
\text { Muestreo }\end{array}$ & Muestreo de Conveniencia. & Muestreo de Conveniencia. \\
\hline Error Muestral & $\begin{array}{l}+/-4 \% \text { para un intervalo de confi- } \\
\text { anza del } 95 \% \text { bajo la hipótesis de } \\
\qquad \mathrm{p}=\mathrm{q}=0,5\end{array}$ & $\begin{array}{l}+/-4 \% \text { para un intervalo de confianza } \\
\text { del } 95 \% \text { bajo la hipótesis de } p=q=0,5\end{array}$ \\
\hline $\begin{array}{l}\text { Técnica de recogida } \\
\text { de información }\end{array}$ & $\begin{array}{c}\text { Encuesta personal administrada a } \\
\text { los emprendedores/comerciantes } \\
\text { del área comercial de barrio Italia } \\
\text { a través de un cuestionario estruc- } \\
\text { turado. }\end{array}$ & $\begin{array}{l}\text { Encuesta personal administrada a los } \\
\text { emprendedores/comerciantes del área } \\
\text { comercial de barrio Italia a través de } \\
\text { un cuestionario estructurado. }\end{array}$ \\
\hline $\begin{array}{l}\text { Período de recogida } \\
\text { de información }\end{array}$ & Noviembre-Diciembre 2015. & Abril -Mayo de 2017. \\
\hline $\begin{array}{l}\text { Tratamiento de la } \\
\text { información }\end{array}$ & $\begin{array}{c}\text { Análisis univariable y bivariable } \\
\text { descriptivo. }\end{array}$ & $\begin{array}{c}\text { Análisis univariable y bivariable } \\
\text { descriptivo }\end{array}$ \\
\hline $\begin{array}{l}\text { Procesamiento de la } \\
\text { información }\end{array}$ & Dyane, Excel, SPSS 12.0. y Gephi. & $\begin{array}{c}\text { Dyane, Excel, SPSS 12.0. y UCI- } \\
\text { NET. }\end{array}$ \\
\hline
\end{tabular}

Fuente: Elaboración propia. 


\section{RESULTADOS}

Son las ciudades más expuestas al turismo masivo las primeras interesadas en descongestionar sus zonas históricas y promocionar nuevos barrios bajo marcas de garantía como "microdestinos" dentro de las propias ciudades, tal como está ocurriendo con el Barrio de Las Letras de Madrid o en Barrio Italia en Santiago de Chile (véase figura 1).

Figura 1: Generación de marca en Barrio de las Letras (Madrid) y Barrio Italia (Chile). Marca utilizada como nombre de marca del barrio y como distintivo de los comercios de dichos barrios.
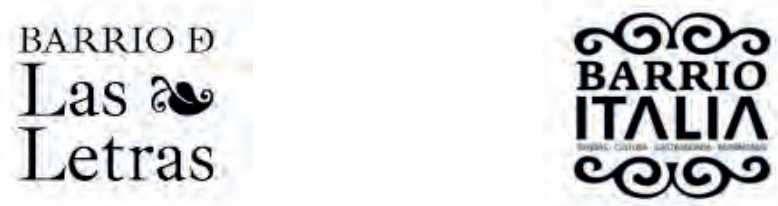

Fuente: Elaboración propia.

En el sector turístico una marca destino es el nombre del lugar y dichos destinos han de trabajar con el fin de crear una imagen de marca positiva e identificativa del lugar.

En el caso del Barrio de Las Letras el nombre identifica, claramente, el origen literario de dicho barrio, donde vivieron escritores como Cervantes o Lope de Vega, creando así una identidad e imagen basada en el origen histórico y literario de la zona.

En el caso de Barrio Italia, la marca se identifica con la historia del lugar. Es un barrio de antiguas edificaciones, donde vivieron artesanos inmigrantes de diferentes países, ante todo italianos, que generaron un activo comercio entre los mismos residentes. La construcción de la fábrica de sombreros Girardi, en 1905, fue uno de los hechos más relevantes en la historia del barrio, cuando una familia italiana del mismo nombre se instaló en el sector y dio empleo a cientos de trabajadores que decidieron vivir cerca de la planta, casas comunes en torno a patios de vecinos (conventillos), donde, actualmente, se encuentran ubicados anticuarios, restaurantes, comercios artesanos y galerías de arte. 
La creación de las marcas Barrio de Las Letras y Barrio Italia conciben los barrio/destino como una marca, buscando la consecución de los siguientes objetivos, tal como analizaban Ruiz, Olarte e Iglesias (1999):

- Identificación de la zona o territorio o barrio de Madrid y Santiago de Chile (véase figura 2, donde se percibe la clara delimitación de los barrios)

- Utilidad de selección o elección por parte de los usuarios: sus nombres se identifican con un barrio histórico y literario (Barrio de Las Letras) y un barrio histórico relacionado con la tradición de la comunidad italiana ubicada en el mismo relacionada con el comercio (Barrio Italia).

- Garantía: la marca representa una expectativa de recursos a encontrar por parte de los posibles consumidores. En el caso de Barrio de Las Letras se trata de recursos relacionados con la literatura, el arte, la historia y el comercio tradicional y en el caso de Barrio Italia se trata de recursos relacionados con la historia, las antigüedades, el comercio tradicional, los patios antiguos donde se encuentran los negocios y la oferta gastronómica.

- Valor de personalización: este valor se consigue en los dos barrios gracias a la existencia de pequeños comercios, anticuarios, galerías de arte, pequeños restaurantes que ofrecen productos y servicios personalizados fuera de la estandarización de los servicios masivos y del comercio "main street".

Estos comercios no solo forman parte del patrimonio histórico y cultural de la ciudad, sino que además cada vez adquieren mayor reconocimiento por ser capaces de adaptarse a los nuevos tiempos, sin perder de vista la tradición que los sustenta. Partiendo de esa base y teniendo en cuenta que lo "vintage" y lo "retro" están más de moda que nunca, estos establecimientos tienen actualmente un gran potencial y no cesan en su propósito de ensalzar los valores los comercios con larga vida, esos que permiten hacer un viaje en el tiempo a través de productos, objetos, olores y sabores.

- Valor lúdico: en el caso del Barrio de Las Letras y Barrio Italia se generan experiencias relacionadas con eventos culturales, gastronómicos, artísticos y literarios (véase figura 3 , que recoge algunas de las actividades lúdicas generadas por ambas asociaciones de comerciantes y figura 4, que recoge la información de la página web de las dos Asociaciones de comerciantes) 
Bajo las marcas Barrio de Las Letras y Barrio Italia, se crea una marca cuya finalidad es promocionar el atractivo de un barrio del centro de Madrid y Santiago de Chile e impulsar la actividad cultural, comercial y de ocio del mismo.

Figura 2: Planos de ubicación de Barrio de las Letras y Barrio Italia

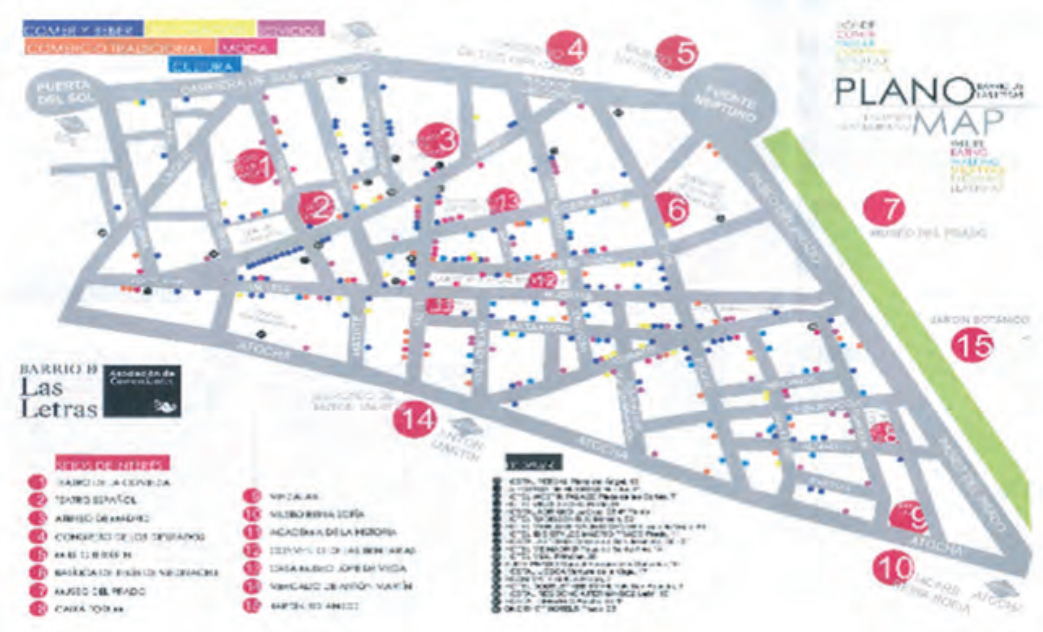

Ubicación de Barrio de las Letras entre Carrera de San Jerónimo, Paseo del Prado, Calle atocha y Plaza del Ángel (Madrid).

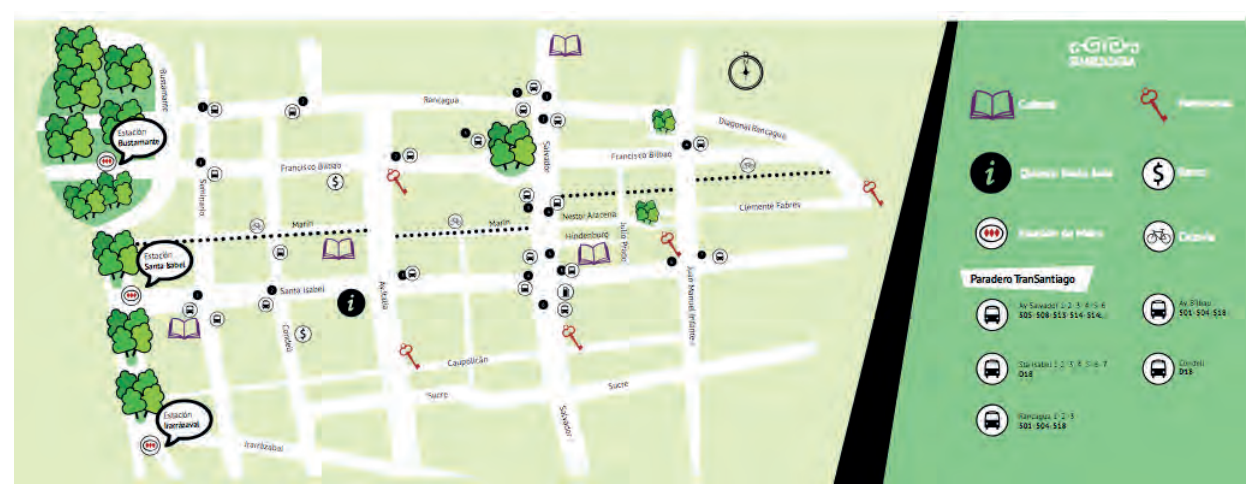

Ubicación de Barrio Italia en la Comuna de Providencia y Ñuñoa (Santiago de Chile), entre Calle Seminario, Rancagua, Sucre y José Manuel Infante.

Fuente: Elaboración propia. www.barrioletras.com http://www.barrioitalia.com/ 
Figura 3: Actividades culturales y lúdicas ofrecidas por Barrio de Las Letras y Barrio Italia para generar experiencias a los consumidores potenciales.
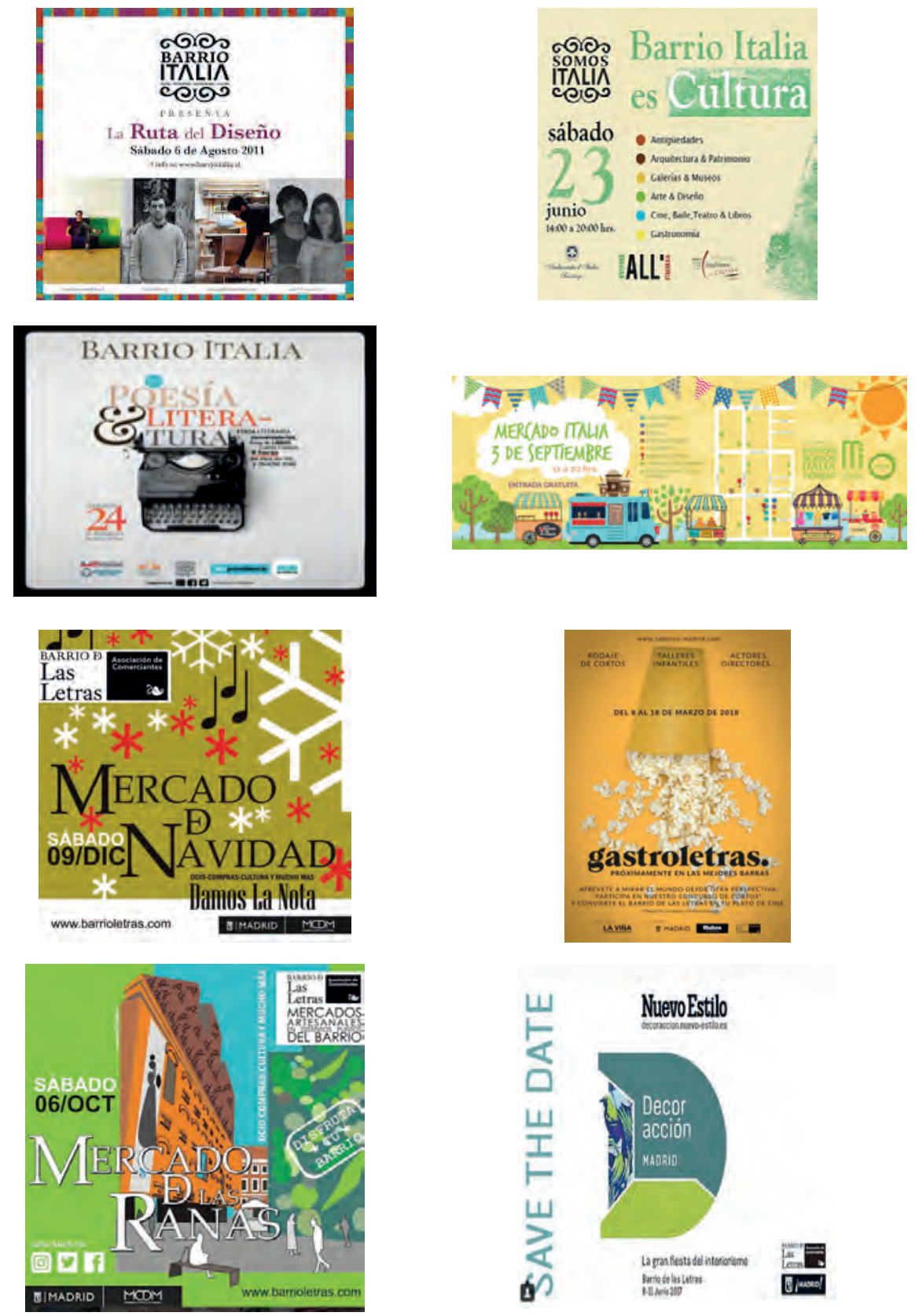

Fuente: Elaboración propia. www.barrioletras.com http://www.barrioitalia.com/ 
Figura 4: Imagen de la página web la Asociación de Comerciantes Barrio de Las Letras y de la Asociación de Comerciantes Barrio Italia, como instrumento de comunicación de las actividades lúdicas ofrecidas.

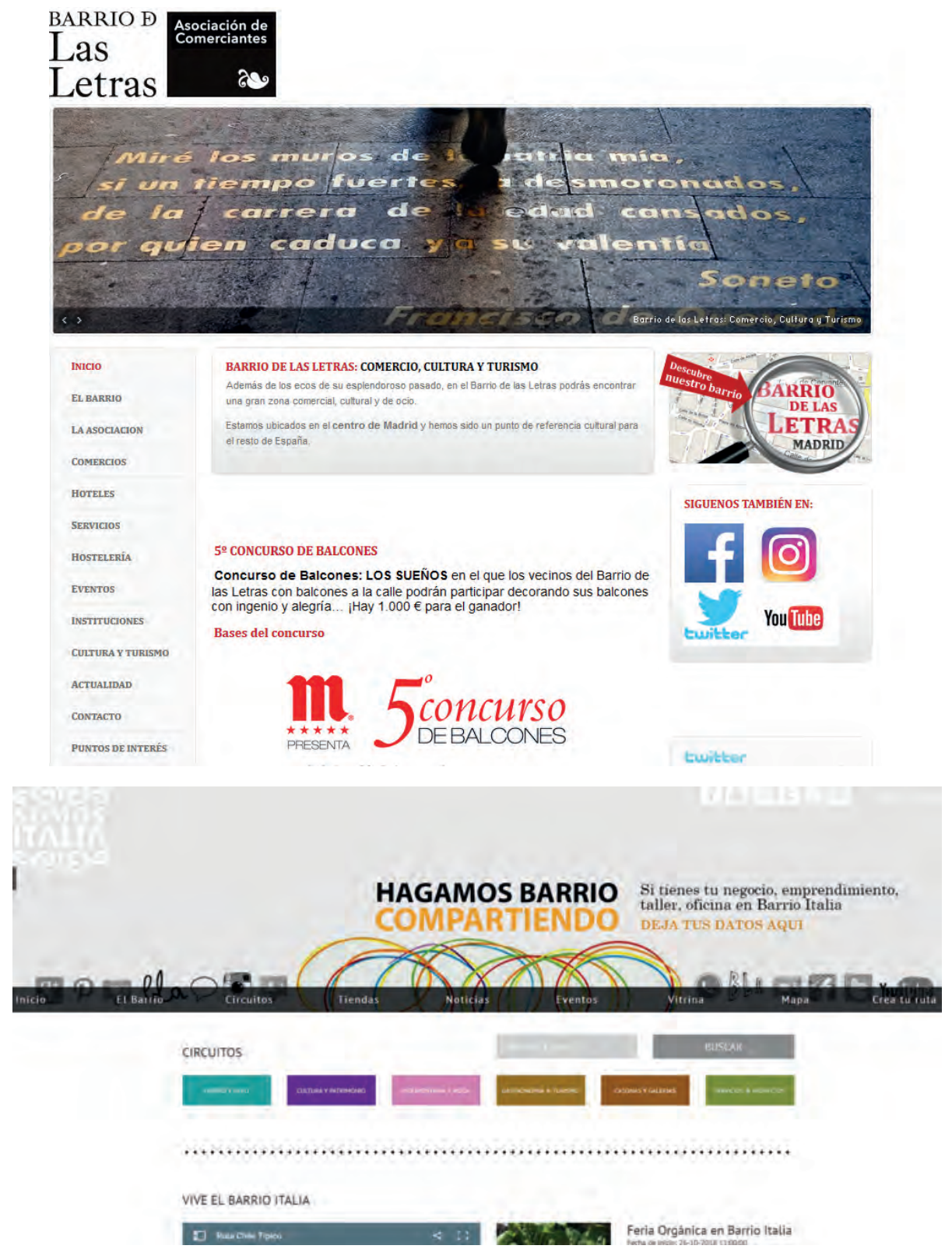

Fuente: http://www.barrioletras.com/ y http://www.barrioitalia.com/ 
La oferta cultural, comercial y de ocio los barrios analizados, supone una adaptación y remodelación de un casco histórico que oferta nuevas experiencias a los turistas con nuevas necesidades y, a todo ello, ha contribuido el asociacionismo y las redes de colaboración del pequeño comercio emprendedor de la zona, junto con instituciones culturales. Dichos comercios son pequeños comercios con aproximadamente tres empleados, comercios de cercanía y marcas propias basadas en la creatividad (Véase tabla 2).

Tabla 2: Perfil del pequeño comercio en Barrio de Las Letras (Madrid) y Barrio Italia (Santiago de Chile).

\begin{tabular}{|c|c|}
\hline BARRIO DE LAS LETRAS (Madrid) & BARRIO ITALIA (Santiago de Chile) \\
\hline Moda $(21,05 \%)$ & Anticuarios (12\%) \\
Arte $(15,79 \%)$ & Servicios gastronómicos (24\%) \\
Tiendas de antigüedades $(9,47 \%)$ & Moda, textil y accesorios $(20 \%)$ \\
Tiendas de alimentación (8,42\%) & Hogar y decoración (12\%) \\
Librerías (6,32\%) & Otros (13\%) \\
Tiendas de decoración (6,32\%) & \\
\hline
\end{tabular}

Fuente: Elaboración propia.

Según los datos extraídos de la tabla, se observa que los establecimientos comerciales que crean la identidad del barrio en su mayoría se asocian con alimentación, moda, textil y accesorios junto con hogar, decoración y antigüedades.

Tanto Barrio de las Letras como Barrio Italia tienen como eje común, su posicionamiento basado en tres ítems principales: comercio, cultura y turismo, resultando ser referentes al momento de hablar de barrios históricos tanto en Chile como en España.

Dichos barrios guardan, además, una amplia riqueza en la existencia de actividad de emprendimiento artístico-cultural, pero en fases de desarrollo opuestas, lo cual resulta mayormente interesante al análisis, tal como se recoge en la figura 5.

Al aplicar el modelo de ciclo de vida del destino al Barrio de las Letras en Madrid y a Barrio Italia en Santiago de Chile, con los parámetros del ciclo de vida de los destinos turísticos (número de visitantes, evolución de la demanda, características de la oferta, campañas de comunicación y estudio de la competencia) se puede afirmar que el Barrio de Las Letras es un destino que se encuentra en fase de crecimiento estabilizado.

Como otros destinos tradicionales, España se enfrenta al reto de mantener su atractivo para el mercado. Según el informe anual de Frontur, el turismo urbano es una apuesta por parte de Turespaña, 
La última encuesta realizada por la empresa pública Madrid Destino (2017) dibuja un perfil del turista que llega a la capital interesado por la cultura, los museos, la gastronomía, la vida nocturna y la hospitalidad española. Por todo ello, desde Madrid Destino, junto con Turespaña, se ha fijado como meta la atracción de nuevos nichos de mercado: un turismo cosmopolita, que quiere empaparse de cultura, historia, tradiciones y que demanda singularidad. Es en esa oferta de cultura, tradición y singularidad donde juegan un papel importante los diferentes barrios históricos de la capital, como el Barrio de Las Letras de Madrid.

Chile, por el contrario, es un destino en las primeras fases del ciclo de vida como destino. Según la modelización propuesta por Butler $(1980,1996,2001)$ se encuentra entre las fases de implicación y de desarrollo a nivel país. Los productos que comercializa, turismo de naturaleza y turismo cultural, se encuentran en las primeras fases de desarrollo, especialmente el turismo cultural y urbano, donde se encuentra la oferta de Barrio Italia en Santiago de Chile.

Figura 5: Fase de ciclo de vida en el desarrollo de los destinos turísticos: Barrio de Las Letras v/s Barrio Italia.

Ciclo de Vida de Destinos Turísticos

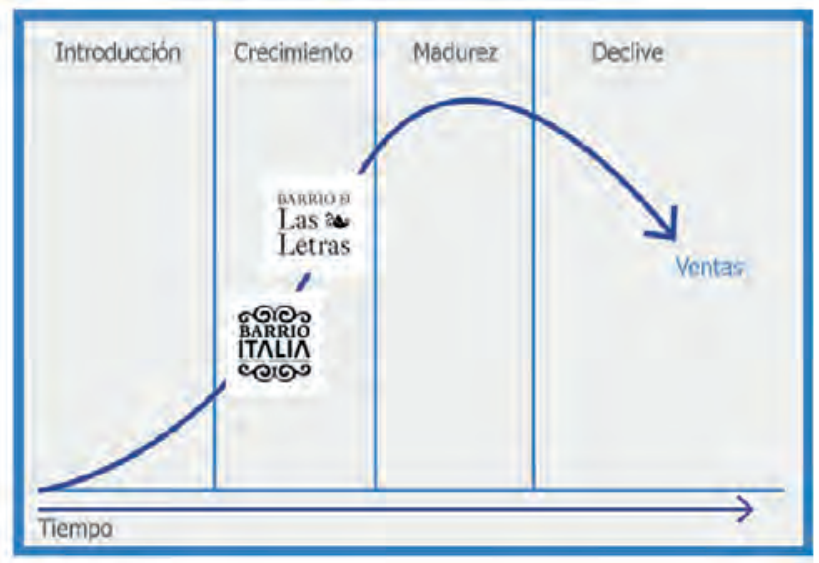

Fuente: Elaboración propia.

Desde la Asociación Barrio de Las Letras se promueven múltiples actividades y campañas de comunicación que muestren a los vecinos, a los visitantes y a los turistas la rica oferta del Barrio de Las Letras. Según el estudio realizado (G.Henche y Salvaj, 2017), las principales acciones comerciales y culturales de los últimos años son el Mercado de Las Ranas (cada primer sábado de mes los comercios sacan su actividad a la calle), Decoacción (evento anual donde anticua- 
rios de toda España toman las calles del Barrio de Las Letras y los negocios e instituciones culturales realizan intervenciones culturales y de diseño) y La Noche de los Libros (actividades en librerías, actos literarios, teatralización en la calle).

En el caso de Barrio Italia se promueven actividades también para darle dinamismo al barrio y hacerlo más interesante, las principales acciones comerciales y culturales de los últimos años son el Mercado Italia, la Ruta del Café y del Té, la Ruta del Vino y la Cerveza, Jazz y Vino (En las calles principales del barrio artistas del jazz comparten su música junto a diversos puestos de viñas pertenecientes al movimiento de viñateros independientes) y Poesía y Literatura (Es una feria literaria con cuentacuentos, firma de libros al aire libre).

\section{DISCUSIÓN}

Timur y Getz (2008) apelan que un desarrollo de turismo urbano está directamente relacionado con una de redes que incorpore y gestione a los stakeholders involucrados, se debe además analizar el vínculo existente entre los cluster de la industria, el gobierno y la comunidad en cuestión. Graci (2013), por su parte, genera un vínculo directo entre desarrollo de turismo sustentable y colaboración y asociacionismo.

La experiencia turística es una actividad de corte psicológico compleja vinculada con el consumidor (Larsen, 2007), las experiencias que se llevan a cabo al visitar un destino quedan en la memoria del consumidor para formar una percepción que se traduce en la imagen del destino, por lo cual el desafío de cada marca de destino viene dado en potenciar experiencias positivas que se puedan traducir en lealtad y preferencias hacia las marcas.

Manejar marcas de destino resulta complejo, para ello Hankinson (2007) desarrolló un modelo de gestión, basado en: “un fuerte liderazgo y visión, una cultura organizacional orientada a las marcas, alineación y coordinación departamental, comunicación fluida entre los diversos stakeholders y fuertes partnerships”.

La cooperación y las redes son un verdadero multiplicador de oportunidades en el sector turístico, creando marca debido a la cooperación de todos los stakeholders involucrados en un área geográfica. La presente investigación exploró cómo la asociación de pequeñas empresas y las redes creadas entre los miembros asociados permiten la transformación de una zona comercial, creando una imagen de destino y aumentando el valor turístico del territorio y de los bienes del barrio, analizando el caso de Barrio Italia, en Santiago de Chile y Barrio de las Letras, Madrid. 


\section{CONCLUSIÓN}

De acuerdo con la aproximación conceptual y la revisión de la literatura realizada en este artículo, es posible concluir que el turismo de experiencias en los barrios históricos adquiere relevancia en el mercado turístico. A su vez, el mercado cada vez más exigente y en busca de experiencias supone un nicho de mercado al que dirigir esas experiencias de dichos barrios históricos y con personalidad

Analizados los resultados de perfiles de emprendedores de Barrio de las Letras y Barrio Italia y de las acciones culturales llevadas a cabo por los mismos, se puede concluir que los ejes principales del trabajo asociativo y de colaboración son el comercio, la cultura y el turismo. Los resultados muestran que se ha conseguido fomentar el desarrollo y sustentabilidad de cada uno de los barrios: un comercio cercano y competitivo, donde la calidad, la profesionalidad y el servicio (atención al cliente) sean las señas de identidad, proteger y poner en valor el comercio tradicional, así como fomentar el asentamiento de nuevas ideas emprendedoras, consolidar el asociacionismo fomentando una cultura de cooperación, articular las bases necesarias para la creación y consolidación de áreas comerciales y culturales urbanas, dotándolas de una estrategia común, así como de una imagen corporativa que refuerce la identidad de los barrios analizados.

El estudio revela el desarrollo de espacios urbanos que tienen como objetivo ofrecer turismo de experiencias generando una ecología de redes y actividades en torno a una marca identificativa del destino, en este caso "Barrio de las Letras" y "Barrio Italia"

La oferta cultural, comercial y de ocio de los barrios objeto de estudio supone una adaptación y remodelación de un casco histórico que oferta nuevas experiencias a los turistas con nuevas necesidades y a todo ello ha contribuido el asociacionismo y las redes de colaboración del pequeño comercio emprendedor de la zona, junto con instituciones culturales ubicadas en dicho barrio.

La regeneración y dinamización de los barrios analizados se consigue gracias al papel de dinamización comercial de los asociados, que consiguen ofrecer un "microdestino" con actividades que permiten al viajero entrar en contacto con el estilo de vida local y con la cultura de los residentes

Analizada la imagen de marca de los dos barrios estudiados se puede afirmar que se ha conseguido articular las bases necesarias para la creación y consolidación de dos Áreas Comerciales Urbanas, dotándolas de una estrategia común, así como de una imagen corporativa que refuerza su identidad. 
El estudio muestra que para conseguir la creación marcas destino se requiere de un trabajo en múltiples dimensiones (plataformas de red, generación de servicios especializados, comercios especializados o de nicho, gastronomía de calidad, hostelería personalizada entre otros); por ello, es fundamental, que exista una red de coordinación entre los comerciantes, lo cual se ve facilitado las dos asociaciones de comerciantes que asumen un papel de liderazgo y coordinación.

La contribución principal del estudio viene dada por la integración de componentes muy diversos para la creación de la imagen de marca de un "barrio destino” y el análisis del uso de esa marca como instrumento de comunicación con el mercado objetivo.

Por otra parte, se percibe una evidente ubicación de cada barrio en fases diferentes de desarrollo en su ciclo de vida como destino turístico, Barrio Italia se encuentra en una fase incipiente de crecimiento frente a Barrio de las Letras que ya cuenta con un crecimiento más consolidado. La investigación abre espacio a posteriores desarrollos en el campo del urbanismo del turismo cultural y aporta directamente a la comunidad científica.

\section{REFERENCIAS BIBLIOGRÁFICAS}

Agapito, D., Mendes, J. y Valle, P. (2013): «Exploring the conceptualization of the sensory dimension of tourist experiences». Journal of Destination Marketing \& Management, 2(2) 62-73.

Agapito, D., Valle, P. y Mendes, J. (2014): «The sensory dimension of tourist experiences: Capturing meaningful sensory-based themes in Southwest Portugal». Tourism Management, 42: 224-237.

Bandarin, F. y Van Oers, R. (2012): «The Historic Urban Landscape: managing heritage in an urban century». Oxford: Wiley-Blackwell.

Barbieri, A., Almeida Santos, C. y Katsube, Y. (2012): «Volunteer tourism: On the ground observations from Rwanda». Tourism Management, 33, 509-516.

Barrio de las Letras (2018): Asociación de Comerciantes del Barrio de Las Letras. En línea: http://www.barrioletras.com, consultado el 25/10/2018

Barrio Italia (2018): Asociación de Comerciantes de Barrio Italia. En línea: http://www. barrioitalia.com, consultado el 25/10/2018.

Bigné Alcañiz, E., Sanchez, M. I. y Sanchez, J. (2001): «Tourism image, evaluation variables and after purchase behavior: Inter-relationship». Tourism Management, 22 (6) 607-616.

Butler, R. (1980): «The Concept of a Tourist Area Cycle of Evolution: Implications for Management of Resources». Canadian Geographer, 24 (1) 5-12. 
Butler, R. (1996): «The Concept of Carrying Capacity for Tourism Destinations: Dead or Merely buried». Progress in Tourism and Hospitality Research, 2 (3-4) 283-293.

Butler, R. (2001): «The Resort Cycle Two Decades On. Tourism in the 21st Century. Lessons from Experience». Chichester: John Wiley \& Sons.

Camarero, C. y Garrido, M.J. (2004): Marketing del Patrimonio Cultural. Ediciones Pirámide, ESIC, Madrid.

Christaller, W. (1964): «Some Considerations of Tourism Location in Europe: The Peripheral Regions- Underdeveloped Countries - Recreation Areas». Papers in Regional Science, 12 (1), 95-105.

Cohen, E. (1972): «Toward a Sociology of International Tourism». Social Research, 39, 164-182.

Gabbay, S.M. y Zuckerman, E. (1998): «Social capital and opportunity in corporate R\&D: The contingent effect on contact density on mobility expectations». Social Science Research, 27 (2) 189-217.

Graci, S. (2013): «Collaboration and Partnership Development for sustainable tourism». Tourism Geographies, 15 (1) 25-42.

García Henche, B. (2017): «Los mercados de abastos y su comercialización como producto de turismo de experiencias. El caso de Madrid». Cuadernos de Turismo, 39, 631-632.

García Henche, B. y Salvaj, E. (2017): «Associations, networks and the transformation toward experiential tourism marketing. The case of the district Barrio de las Letras, Madrid». Cuadernos de Turismo.

Hall, C.M. y Weiler, B. (1992): «Special interest tourism», CABI.

Hallak, R., Brown, G. y Lindsay, N. (2012): «The place identity-Performance relationship among tourism entrepreneurs: A structural equation modelling analysis». Tourism Management, 33, 143-154.

Hankinson, G. (2007): «The management of destination brands: Five guiding principles based on recent developments in corporate branding theory». Journal of Brand Management, 14, 240-254.

Harrison, D. (1995): «Development of Tourism in Swaziland». Annals of Tourism Research, 22 (1) 135-156.

Jun Song, H., Park, J., Hwang, Y. y Reisinger, Y. (2015): «The influence of tourist experience on perceived value and satisfaction with temple stays: The experience economy theory». Journal of Travel \& Tourism Management, 32 (4) 401-415.

Kastenholz, E., Carneiro, M. J., y Marques, C. (2012): «Marketing the rural tourism experience». Strategic Marketing in Tourism Services, 247-264.

King, J. (2002): «Destination marketing organizations - Connecting the experience rather than promoting the place». Journal of Vacation Marketing, 8 (2) 105-108.

Kotler, P. y Gertner, D. (2002): «Country as a brand, product, and beyond: A place marketing and brand management perspective». Brand Management, 9 (4-5) 249-261.

Larsen, S. (2007): «Aspects of psychology of the tourist experience». Scandinavian Journal of Hospitality and Tourism, 7 (1) 7-18.

Lo, A. y Lee, C. (2011): «Motivations and perceived value of volunteer tourism from Hong Kong». Tourism Management, 32, 326-334. 
Lundtorp, S. y Wanhill, S. (2001): «The Resort Lifecycle Theory: Generating Processes and Estimation». Annals of Tourism Research, 28 (4) 947-964.

Madrid Destino (2018): Empresa encargada de la gestión cultural y turística de Madrid. En línea: http://madrid-destino.com/es, consultado el 03/05/22018

McGehee, N. y Andereck, K. (2009): «Volunteer Tourism and the "voluntoured": The case of Tijuana, Mexico». Journal of Sustainable tourism, 17 (1) 39-51.

Mendonça, V., Varajão, J. y Oliveira, P. (2015): «Cooperation Networks in the Tourism Sector: Multiplication of Business Opportunities». Procedia Computer Science, 64, 1172 - 1181.

Molina, A., Martín Consuegra, D., Esteban, A. y Díaz, E. (2007): «Segmentación de la demanda turística: Un análisis aplicado a un destino cultural». Revista de análisis turístico, nº 4, 36-48.

Nahapiet, J. y Ghoshal, S. (1998): «Product country images: Importance and role in International Marketing». International Business Press, New York.

Pine, J. y Gilmore, J. (1998): «Welcome to the Experience Economy». Harvard Business Review.

Plog, S.C. (1974): «Why Destination Areas Rise and Fall in Popularity». The Cornell Hotel and Restaurant Administration Quarterly, 42 (3) 55-58.

Robinson, M. y Novelli, M. (2005): «Niche Tourism». Contemporary issues, trends and cases. Routledge.

Ruiz, A. V., Olarte, R. e Iglesias, V. (1999). «Evaluación de los destinos turísticos en función de su valor de marca. Actas del XI Encuentro de Profesores Universitarios de Marketing», 427-450

Schmitt, B. (1999): «Experiential Marketing». Journal of Marketing Management, 15 (1-3) 53-67.

Serra Cantallops, A. (2011): Marketing Turístico. Editorial ESIC-Pirámide, Madrid.

Timur, S. y Getz, D. (2008): «A network perspective on managing stakeholders for sustainable urban tourism». International Journal of Hospitality Management, 20 (4) 445-461.

Toitiño Vinuesa, M. A. (1991): «Centro histórico, intervención urbanística y análisis urbano», Revista Anales de Geografía de la Universidad Complutense, 11, 25-48.

Vogeler Ruiz, C. y Hernández Armand, E. (2002): El Mercado Turístico: estructura, operaciones y procesos de producción. Editorial Centro de Estudios Ramón Areces, Madrid.

Wearing, S. (2001). Volunteer Tourism. Experiences that make a difference. CABI Publishing.

Wang, R.F. y Simons, D.J. (1999): «Active and passive scene recognition across views». Cognition, 70, 191-210.

Zach, F. y Hill, T.L. (2017): «Network, knowledge and relationship impacts on innovation in tourism destinations». Tourism Management, 62, 196-207. 